\title{
THE EQUIVALENCE OF CERTAIN REGULAR TRANSFORMATIONS*
}

BY

\section{L. SILVERMAN}

1. Introduction. The transformation

$$
v(x)=\boldsymbol{\alpha} u(x)+\int_{0}^{x} k(x, y) u(y) d y,
$$

or symbolically, $v=A(u)$, has been studied in special cases by Landau, $\uparrow$ and in the general case by the author. $\neq$ It is assumed that $u(x)$ is bounded and integrable, $x_{1} \geqq x \geqq 0$, that $k(x, y)$ is integrable in $y$ for each positive $x$, $0<\delta \leqq y \leqq x$, and that $\int_{0}^{x}|k(x, y)| d y$ converges for each positive $x$. The function $k(x, y)$ will be called the kernel and the symbol $[\alpha, k(x, y)]$ the matrix of the transformation. The transformation is regular if the existence of $\lim _{x \rightarrow \infty} u(x)$ implies the existence of $\lim _{x \rightarrow \infty} v(x)$ and the equality of the limits. Examples of regular transformations are

$$
E:[1,0], \quad M:\left[0, \frac{1}{x}\right],
$$

the transformations of Cesäro of order $r$,

$$
C_{r}:\left[0, \frac{r}{x}\left(1-\frac{y}{x}\right)^{r-1}\right]
$$

and the transformations of Hölder of order $r$,

$$
H_{r}:\left[0, \frac{1}{\Gamma(r)} \frac{1}{x}\left(\log \frac{x}{y}\right)^{r-1}\right] .
$$

For $r=1$, we have $C_{1} \equiv H_{1} \equiv M$.

- Presented to the Society, April 26, 1919, under the title Regular transformations of divergent series and integrals.

† Sächsische Berichte, vol. 65 (1913), p. 131.

¥ These Transactions, vol. 17 (1916), p. 284 ; Annals of Mathematics, ser. 2, vol. 21 (1919), p. 128. 
A sufficient condition* for regularity is

$$
\begin{gathered}
\int_{0}^{a}|k(x, y)| d y \text { converges, } a>0, \\
\lim _{x \rightarrow \infty} \int_{0}^{a}|k(x, y)| d y=0,
\end{gathered}
$$

(3)

(iii)

$$
\begin{gathered}
\int_{0}^{x}|k(x, y)| d y<N, \\
\lim _{x \rightarrow \infty} \int_{0}^{x} k(x, y) d y=1-\alpha .
\end{gathered}
$$

In a previous papert the author has discussed a special matrix in terms of a function of a complex variable $z$, and has obtained conditions for the regularity and equivalence of certain transformations in terms of the corresponding functions of $z$. In order to avoid the use of complex numbers it is proposed to define the matrix $[\alpha, k(x, y)]$ in terms of the real function $f(x)$ satisfying the following conditions:

$$
f(x) \text { is continuous, } 0 \leqq x \leqq 1
$$

$$
f^{\prime}(x) \text { is continuous, } 0<x \leqq 1 \text {, }
$$

(4) (iii)

$$
\begin{gathered}
f(0)=0, \\
\int_{0}^{1}\left|f^{\prime}(x)\right| d x \text { converges. }
\end{gathered}
$$

A function satisfying these conditions will be called absolutely regular. We then define

$$
\alpha=1-f(1), \quad k(x, y)=\frac{1}{x} f^{\prime}\left(\frac{y}{x}\right)
$$

* These Transactions, vol. 17 (1916), p. 292.

† Annals of Mathematics, loc. cit., p. 134. 
so that solving for the function $f(x)$, we have

$$
f(1)=1-\alpha, \quad f(x)=\int_{0}^{x} k(1, s) d s .
$$

We now state the theorem

THEOREM 1. If the function $f(x)$ is absolutely regular, then the corresponding transformation is regular.

To prove this theorem, define $\Phi(x)=\int_{0}^{x}\left|f^{\prime}(t)\right| d t, 0<x \leqq 1 ; \boldsymbol{\Phi}(0)=0$. Then $\boldsymbol{\Phi}(x)$ is continuous, $0 \leqq x \leqq 1$. We now obtain from conditions (3)

$$
\begin{aligned}
\int_{0}^{a}|k(x, y)| d y & =\int_{0}^{a / x}\left|f^{\prime}(s)\right| d s=\Phi\left(\frac{a}{x}\right), \\
\lim _{x \rightarrow \infty} \int_{0}^{a}|k(x, y)| d y & =\lim _{x \rightarrow \infty} \Phi\left(\frac{a}{x}\right)=\Phi(0)=0, \\
\int_{0}^{x}|k(x, y)| d y & =\int_{0}^{1}\left|f^{\prime}(s)\right| d s=\Phi(1), \\
\int_{0}^{x} k(x, y) d y & =\int_{0}^{1} f^{\prime}(s) d s=f(1)=1-\alpha .
\end{aligned}
$$

Thus the theorem is proved.

2. The product transformation. We now consider three transformations $A, B, C$ corresponding to the absolutely regular functions $f(x)$, $g(x), h(x)$, and inquire as to the form of $h(x)$ when $C=B A$. We have

(A)

$$
v(x)=\alpha u(x)+\int_{0}^{x} k(x, y) u(y) d y
$$

$$
=u u(x)+\int_{0}^{1} f^{\prime}(s) u(x s) d s
$$




$$
\begin{aligned}
w(x) & =\beta v(x)+\int_{0}^{1} g^{\prime}(t) v(x t) d t \\
& =\alpha \beta u(x)+\beta \int_{0}^{1} f^{\prime}(s) u(x s) d s+\alpha \int_{0}^{1} g^{\prime}(s) u(x s) d s \\
& +\lim _{\varepsilon \rightarrow 0} \int_{\varepsilon}^{1} \int_{0}^{1} f^{\prime}(s) g^{\prime}(t) u(x s t) d s d t .
\end{aligned}
$$

Now letting $t s=y$ in the double integral, and then using the abbreviations

$$
\begin{aligned}
& F(y, t)=\frac{1}{t} g^{\prime}(t) f^{\prime}\left(\frac{y}{t}\right) u(x y), \\
& \boldsymbol{\Phi}(\delta, t)=\int_{\delta}^{t} F(y, t) d y,
\end{aligned}
$$

the double integral becomes

$$
\lim _{\varepsilon \rightarrow 0} \int_{\varepsilon}^{1} \lim _{\delta \rightarrow 0} \boldsymbol{\Phi}(\delta, t) d t .
$$

Now holding $\varepsilon$ fixed, and letting $P$ be an upper bound of $|u(x)|$, and $Q$ the maximum of $\left|g^{\prime}(t)\right|$ in the interval $(\varepsilon, 1)$, we have

$$
\begin{aligned}
|\boldsymbol{\Phi}(\delta, t)-\boldsymbol{\Phi}(0, t)| & \leqq \int_{0}^{\delta}|F(y, t)| d y<P Q \int_{0}^{\delta} \frac{1}{t}\left|f^{\prime}\left(\frac{y}{t}\right)\right| d y \\
& =P Q \int_{0}^{\delta / t}\left|f^{\prime}(s)\right| d s \leqq P Q \int_{0}^{\delta / \varepsilon}\left|f^{\prime}(s)\right| d s<\varepsilon_{1}
\end{aligned}
$$

provided $\delta<\delta_{1}$, where $\delta_{1}$ is independent of $t$. Hence $\lim _{\delta \rightarrow 0} \Phi(\delta, t)$ exists uniformly in $t, \varepsilon \leqq t \leqq 1$, and 


$$
\int_{\varepsilon}^{1} \lim _{\delta \rightarrow 0} \Phi(\delta, t) d t=\lim _{\delta \rightarrow 0} \int_{\varepsilon}^{1} \Phi(\delta, t) d t
$$

so that (7) becomes

$$
\begin{aligned}
\lim _{\varepsilon \rightarrow 0} \lim _{\delta \rightarrow 0} \int_{\varepsilon}^{1} \int_{\delta}^{t} F(y, t) d y d t & \\
= & \lim _{\delta \rightarrow 0} \int_{\delta}^{1} \int_{\delta}^{t} F(y, t) d y d t+\lim _{\delta \rightarrow 0} \lim _{\delta \rightarrow 0} \int_{\delta}^{\delta} \int_{\delta}^{t} F(y, t) d y d t .
\end{aligned}
$$
Now the second term of the last expression is zero; for, letting $\int_{0}^{1}\left|f^{\prime}(s)\right| d s=R$,
we have

$$
\begin{aligned}
\left|\int_{\varepsilon}^{\delta} \int_{\delta}^{t} F(y, t) d y d t\right| & \leqq P \int_{\varepsilon}^{\delta} \int_{\delta}^{t}\left|\frac{g^{\prime}(t)}{t} f^{\prime}\left(\frac{y}{t}\right)\right| d y d t \\
& =P \int_{\varepsilon}^{\delta} \int_{\delta / t}^{1}\left|g^{\prime}(t) f^{\prime}(s)\right| d s d t<P \int_{\varepsilon}^{\delta} \int_{0}^{1}\left|g^{\prime}(t) f^{\prime}(s)\right| d s d t \\
& =P R \int_{\delta}^{\delta}\left|g^{\prime}(t)\right| d t,
\end{aligned}
$$

and the last expression approaches zero*, as $\delta$ and $\varepsilon$ both approach zero. Hence (7) reduces to

$$
\lim _{\delta \rightarrow 0} \int_{\delta}^{1} \int_{\delta}^{t} F(y, t) d y d t=\lim _{\delta \rightarrow 0} \int_{\delta}^{1} \int_{y}^{1} F(y, t) d t d y
$$

* It has been assumed that $\delta \geqq \varepsilon$; the case $\delta<\varepsilon$ would produce only a change in sign in the preceding inequalities and lead to the same conclusion. 
and returning to $B$ we may write

$$
\begin{aligned}
w(x)=\alpha \beta u(x)+\beta \int_{0}^{1} f^{\prime}(s) u(x s) d s & +\alpha \int_{0}^{1} y^{\prime}(s) u(x s) d s \\
& +\int_{0}^{1}\left[\int_{s}^{1} \frac{g^{\prime}(t)}{t} f^{\prime}\left(\frac{s}{t}\right) d t\right] u(x s) d s .
\end{aligned}
$$

Identifying this with

$$
w(x)=\gamma u(x)+\int_{0}^{1} h^{\prime}(s) u(x s) d s,
$$

we have

$$
\gamma=\alpha \beta, \text { or } h(1)=f(1)+g(1)-f(1) g(1),
$$

$$
h^{\prime}(x)=\beta f^{\prime}(x)+\alpha g^{\prime}(x)+\int_{x}^{1} \frac{g^{\prime}(t)}{t} f^{\prime}\left(\frac{x}{t}\right) d t .
$$

Letting $x / t=s$, we find $^{*}$

$$
h^{\prime}(x)=\alpha g^{\prime}(x)+\beta f^{\prime}(x)+\int_{x}^{1} \frac{f^{\prime}(s)}{s} g^{\prime}\left(\frac{x}{s}\right) d s .
$$

Hence we see that the transformations $A$ and $B$ are permutable, that is, $A B=B A$. It follows from this that all absolutely regular transformations are consistent. $\ddagger$

Returning to (9), we have

$$
h(x)=\int_{0}^{x} h^{\prime}(t) d t=\beta f(x)+\alpha g(x)+\int_{0}^{x} \int_{t}^{1} \frac{g^{\prime}(s)}{s} f^{\prime}\left(\frac{t}{s}\right) d s d t .
$$

* Cf. Nielsen, Handbuch der Theorie der Gammafunktion, p. 119.

$\dagger$ ProfessorW.A.Hurwitz has proved and communicated to me the theorem that a necessary and sufficient condition that the transformations $A$ and $M$ of $\S 1$ be permutable is that the kernel of $A$ be homogeneous of degree -1 .

$\ddagger$ Hurwitz and Silverman, these Transactions, vol. 18 (1917), p. 7. 
Now

$$
\int_{t=0}^{x} \int_{s=t}^{1}=\int_{t=0}^{1} \int_{s=t}^{1}-\int_{t=x}^{1} \int_{s=t}^{1}=\int_{s=0}^{1} \int_{t=0}^{s}-\int_{s=x}^{1} \int_{t=x}^{s},
$$

whence

$$
h(x)=[1-g(1)] f(x)+g(x)+\int_{x}^{1} f\left(\frac{x}{s}\right) g^{\prime}(s) d s
$$

Similarly

$$
h(x)=[1-f(1)] g(x)+f(x)+\int_{x}^{1} g\left(\frac{x}{s}\right) f^{\prime}(s) d s
$$

Letting $x==1$ in either (11) or (12) we obtain (8).

Suppose now that $f(x)$ and $g(x)$ are absolutely regular; then $h(x)$ also is absolutely regular. For $h(x)$ clearly satisfies the second and third conditions of (4). As to the fourth condition, we have

$$
\begin{aligned}
\int_{\varepsilon}^{1}\left|h^{\prime}(x)\right| d x \leqq|\beta| \int_{\varepsilon}^{1}\left|f^{\prime}(x)\right| d x+|\alpha| & \int_{\varepsilon}^{1}\left|g^{\prime}(x)\right| d x \\
& +\int_{\varepsilon}^{1} \int_{x}^{1} \frac{1}{t}\left|g^{\prime}(t) f^{\prime}\left(\frac{x}{t}\right)\right| d t d x .
\end{aligned}
$$

Now, interchanging the order of integration in the double integral, making the substitution $x=y t$ in that integral, and letting $R=\int_{0}^{1}\left|f^{\prime}(s)\right| d s$, we obtain for the value of the double integral,

$$
\int_{\varepsilon}^{1}\left|g^{\prime}(t)\right| \int_{\varepsilon / t}^{1}\left|f^{\prime}(y)\right| d y<\int_{\varepsilon}^{1}\left|g^{\prime}(t)\right| \int_{0}^{1}\left|f^{\prime}(y)\right| d y d t=R \int_{\varepsilon}^{1}\left|g^{\prime}(t)\right| d t
$$

hence $\int_{0}^{1}\left|h^{\prime}(x)\right| d x$ converges. Finally, since $\int_{0}^{x}\left|h^{\prime}(t)\right| d t$ is continuous, $0 \leqq x \leqq 1$, it follows that $h(x)$ is continuous, $0 \leqq x \leqq 1$. Thus $h(x)$ is absolutely regular. 
We shall now define an absolutely regular transformation as one which corresponds to an absolutely regular function, and collect the results of this section in the following statement:

THEOREM 2. If the functions $f(x), g(x), h(x)$ correspond to the transformations $A, B, C$ respectively, and if $C=A B$, then $h(x)$ is given in terms of $f(x)$ and $g(x)$ by either of the formulas (11), (12). If $f(x)$ and $g(x)$ are absolutely regular, then $h(x)$ also is absolutely regular. All absolutely regular transformations are permutable and hence consistent.

3. Inclusion and equivalence. We shall hereafter denote by the symbol $f$ the transformation corresponding to the function $f(x)$. The transformation $f$ is said to include the transformation $g$ whenever $f$ evaluates the limit of any function evaluated by $g$, and to the same value; in symbols, $f \geqq g$. If each of two transformations includes the other, they are called equivalent and we write* $f=g$.

We shall now show that in order that the transformation $h$ include the transformation $g$ it is sufficient that there exist a solution $f(x)$ of the integral equation (11) or (12) which is absolutely regular. To see this, let $u(x)$ be any function transformed by $h$ into $v(x)$ and by $g$ into $w(x)$; i. e., $h(u)=v$ and $g(u)=w$. Then $f(w)=f g(u)$. But by hypothesis $f g=h$; hence $f(w)=h(u)=v$. It is now clear that, since $f$ is regular, $\lim _{x \rightarrow \infty} v(x)=\xi$ whenever $\lim _{x \rightarrow \infty} w(x)=\xi$; in other words $h \geqq g$. Abbreviating equations (11), (12), by the symbols

$$
\begin{aligned}
& h(x)=g(x) \circ f(x), \\
& h(x)=f(x) \circ g(x),
\end{aligned}
$$

we may say

THEOREM 3. A sufficient condition that the transformation $f$ include the transformation $g$ is that there exist an absolutely regular solution $p(x)$ of the integral equation $f(x)=p(x) \circ g(x)$.

CoRollary 1. A sufficient condition that the transformations $f$ and $g$ be equivalent is that there exist two absolutely regular functions $p(x), q(x)$ satisfying the equations $f(x)=p(x) \circ g(x), g(x)=q(x) \circ f(x)$.

COROLlary 2. A sufficient condition that the transformation $f$ be equivalent to the identical transformation $E$ is that there exist an absolutely regular solution of the equation $f(x) \circ p(x)=0$.

4. Examples.

(i) The function corresponding to the transformation of Cesàro of order $r$ is $C_{r}(x)=1-(1-x)^{r}$.

This follows directly from (1) and (6).

\footnotetext{
* The notation $f \equiv g$ will indicate the identity of $f$ and $g$.
} 
(ii) The function corresponding to the transformation of Holder of order $r$ is

$$
h_{r}(x)=\frac{1}{\Gamma(r)} \int_{0}^{x}\left(\log \frac{1}{t}\right)^{r-1} d t
$$

This follows directly from (2) and (6).

(iii) The transformations of Cesàro and Hölder of order 2 are* equivalent.

To prove this we shall prove the existence of two absolutely regular functions $p(x), q(x)$ satisfying the conditions $c_{2}(x)=h_{2}(x) \circ p(x), h_{2}(x)$ $=C_{8}(x) \circ q(x)$. To determine $p(x)$ we have the equation

$$
1-(1-x)^{2}=x-x \log x+\int_{x}^{1} p\left(\frac{x}{s}\right) \log \frac{1}{s} d s .
$$

Making in the integral the substitution $t=x / s$, dividing by $x$, differentiating both sides with respect to $x$, multiplying by $x$, and again differentiating both sides with respect to $x$, we obtain $p(x)=-x^{2}$, which is obviously absolutely regular.

To determine $q(x)$ we have the equation

$$
x-x \log x=1-(1-x)^{2}+2 \int_{x}^{1} q\left(\frac{x}{s}\right)(1-s) d s .
$$

Making the substitution $t=x / s$, dividing by $x$ and differentiating both sides twice with respect to $x$ we obtain $q(x)=x / 2$, which is obviously absolutely regular.

(iv) The functions

$$
f(x)=a x^{n}, g(x)=\frac{a}{a-1} x^{\frac{n}{1-a}}, \quad n>0, a<1
$$

correspond to transformations equivalent to convergence and hence equivalent to each other. For

$$
\begin{aligned}
f(x) \circ g(x) & =\frac{a}{1-a} x^{n}-\frac{a}{1-a} x^{n /(1-a)}-\frac{n a^{2}}{(1-a)^{2}} \int_{x}^{1}\left(\frac{x}{s}\right)^{n} s^{n /(1-a)-1} d s \\
& =0 .
\end{aligned}
$$

* The method here employed can be used also to deduce the well known theorem concerning the equivalence of these transformations for any integral order $r$. The case of non-integral order will be deduced more readily from a test for equivalence ton be published in a later paper. 
Now if $n>0, a<1$, both functions are absolutely regular, hence our statement is proved.

(v) The transformation* $\alpha E+(1-\alpha) M$ is reversible (equivalent to convergence) when $\alpha>0$.

This follows directly from example (iv), putting $n=1, \alpha=1-a$.

5. Transformation of sequences. The results which we have obtained in the preceding sections for the transformations of functions hold also for transformations of sequences. But inasmuch as a very general treatment of this subject has recently been given by Hausdorff, $t$ we shall consider it only briefly. It should be stated, however, that while Hausdorff devotes some attention to the question of inclusion and equivalence, he does not consider, as we do, the general question of the inclusion and equivalence of absolutely regular transformations.

Let us consider the transformation

$$
y_{n}=\sum_{k=1}^{n} a_{n k} x_{k} \quad(n=1,2,3, \ldots)
$$

where the matrix $\left(a_{n k}\right), k \leqq n$, is a triangular matrix. A sufficient condition that two such transformations be permutable is $\ddagger$ that their matrices be expressible in the form

$$
a_{n k}=\sum_{h=k}^{n}(-1)^{h-k} \frac{(n-1) !}{(n-h) !(h-k) !(k-1) !} a_{h h} .
$$

We shall restrict ourselves to matrices of this form and we shall define $a_{n n}$ in terms of an absolutely regular function $f(x)$,

$$
a_{n n}=1-f(1)+\int_{0}^{1} t^{n-1} f^{\prime}(t) d t .
$$

The transformation so defined is regular§ and will be called absolutely regular. Hausdorff considers the more general case where $f(x)$ is a function with

*I. Schur, Mathematische Annalen, vol. 74 (1913), p. 447; Landau, Sächsische Berichte, vol. 65, p. 131.

† Mathematische Zeitschrift, vol. 9 (1921), p. 74 and p. 280.

†These Transactions, vol. 18 (1917), p. 7.

§ Hausdorff, loc. cit., p. 84. 
limited total fluctuation and $\int_{0} t^{n-1} d f(t)$ is a Stieltjes integral. To obtain the function $h(x)$ corresponding to the product $\left(c_{n k}\right)$ of two transformations $\left(a_{n k}\right)$ and $\left(b_{n k}\right)$ we need only compute $c_{n n}$. If $f(x), g(x)$ correspond to $\left(a_{n k}\right)$, $\left(b_{n k}\right)$, we find $c_{n n}=a_{n n} b_{n n}$, or

$$
\gamma+\int_{0}^{1} t^{n-1} h^{\prime}(t) d t=\left(\alpha+\int_{0}^{1} t^{n-1} f^{\prime}(t) d t\right)\left(\beta+\int_{0}^{1} t^{n-1} g^{\prime}(t) d t\right)
$$

where $\alpha=1-f(1), \beta=1-g(1), \gamma=1-h(1)$. Hence we must determine a function $h(x)$ such that

$$
\gamma=\alpha \beta, \text { i. e., } h(1)=f(1)+g(1)-f(1) g(1),
$$

and

$$
\int_{0}^{1} t^{n-1} h^{\prime}(t) d t=\alpha \int_{0}^{1} t^{n-1} g^{\prime}(t) d t+\beta \int_{0}^{1} t^{n-1} f^{\prime}(t) d t
$$

$$
+\int_{0}^{1} \int_{0}^{1} s^{n-1} t^{n-1} f^{\prime}(s) g^{\prime}(t) d s d t
$$

But such a function $h(x)$ is precisely $h(x)$ as given by formulas (11), (12). For, multiplying (10) by $x^{n-1}$ and integrating from 0 to 1 , interchanging the order of integration* in the resulting double integral, and then in that integral making the substitution $t=x / s$, we obtain precisely (18). Furthermore, letting $x=1$ in (12), we see that (17) also is satisfied. Finally (18) cannot have more than one solution, for if $h_{1}(x)$ is a second solution, we should have

$$
\int_{0}^{1} t^{n-1}\left(h^{\prime}(t)-h_{1}^{\prime}(t) d t=0\right.
$$

* The justification for the interchange of the order of integration can be made as on page 105. 
and hence, by a theorem due to Lerch* and Stieltjes, $t h_{1}^{\prime}(t)=h^{\prime}(t)$. And since $h_{1}(0)=h(0)=0$, it follows that $h_{1}(x)=h(x)$.

It has now been shown that the results of articles 1-3 hold also for sequences.

6. Conclusion. We have obtained two results in this paper. First we have defined the matrix of a transformation for both sequences and functions in terms of an absolutely regular function, and have then obtained the same formula for the function corresponding to the product of two transformations in the case of both sequences and functions. Secondly, we have obtained definite criteria (the same criteria for both sequences and functions) for the inclusion and equivalence of absolutely regular transformations. In another paper we shall consider some further criteria for the inclusion and equivalence of these transformations.

* Rozpravy ceské Akademie, 2d class, vol. 1 (1892), vol. 2 (1893).

†Correspondence d'Hermite et de Stieltjes, vol. 2, pp. 337-339.

Dartmouth College,

HANOVER, N. H. 